ULTIMA DÉCADA N`18, CIDPA VIÑA DEL MAR, ABRIL 2003, PP. 199-227.

\title{
COMPORTAMIENTO DE LAS TRAYECTORIAS EDUCACIONALES Y LABORALES EN JOVENES ESTUDIANTES
}

\author{
ASTRID OYARZÚN* \\ RAÚL IRRAZABAL ${ }^{* *}$
}

LO QUE SE PRESENTA a continuación es una perspectiva analítica de los resultados del estudio realizado por el Área Joven y Educación de CIDPA, por encargo del Programa Chile Califica del Ministerio de Educación. El estudio fue aplicado en 63 centros educativos de las regiones IV, V, VII, VIII, IX y Metropolitana; contemplando la participación de establecimientos educacionales de enseñanza media subvencionados, centros educacionales integrales de adultos (CEIA) y centros de formación técnica (CFT). El universo del estudio fue de 6.301 jóvenes, los que participaron en la aplicación de los instrumentos de carácter cuantitativo y cualitativo. Los resultados de este estudio son un insumo de información que desea responder a la pregunta cómo la institucionalidad pública puede colaborar a un mejor posicionamiento de los jóvenes en sus perspectivas integracionales, desde los sistema de orientación de los liceos de enseñanza media.

El foco de estudio, se centró en la caracterología de las trayectorias educativas y laborales de jóvenes estudiantes, que permitiera, por un lado, establecer los factores que están influyendo en el desarrollo de las trayectorias educativas y laborales de los sujetos: factores

* Asistente Social, Área Joven y Educación, cIDPA. E-Mail: astrid@cidpa.cl.

** Profesor, Área Joven y Educación, cIDPA. E-Mail: raul@cidpa.cl. 
institucionales — sistema educacional, sistema laboral, la política social, la familia, el contexto político- y factores personales —el sí mismo, recursos educacionales y laborales y el capital social-. Y permitiera por otro lado la identificación de tipos de trayectorias de vida en sujetos, que en condiciones semejantes, transitan por trayectorias exitosas o fallidas.

\section{TRAYECTORIAS EDUCACIONALES Y LABORALES}

En la realidad chilena, la variable que más discrimina y determina los pasos siguientes en cuanto al universo posible de trayectorias a seguir por los jóvenes es la dimensión educativa y nivel de escolaridad alcanzado. Utilizando una adecuación de la clasificación de tipos de trayectorias juveniles referenciadas por Bois-Reymond et al. (2002), podemos visualizar cuatro grupos de colectivos de jóvenes de acuerdo a sus logros educacionales y perspectivas de inserción laboral futura (Dávila, 2002:106-108).

i) Trayectorias directas/precarias. Quienes han abandonado o desertado de la escuela, ya sea en la primaria o en la secundaria, no han completado los 12 años de escolaridad, y sus posibilidades de inserción laboral están determinadas a empleos sin calificación, bajos ingresos y precarias condiciones laborales, con elevadas tasas de desempleo. Se ubican principalmente en el sector informal de la economía, sin coberturas de seguridad social ni de salud, con un inicio en la vida laboral a temprana edad.

ii) Trayectorias directas/precarias y semicualificadas. Quienes alcanzan 12 ó 13 años de escolaridad y acceden a una situación de empleo inestable, con alto riesgo de desempleo, que les otorgan elevados niveles de incertidumbre futura, principalmente por la degradación del factor educativo y de los diplomas obtenidos en tiempo presente. Tienen a su favor competir con los bajos niveles de escolaridad de la población adulta y trabajadora.

iii) Trayectoria entre semicualificadas y cualificadas. Quienes alcanzan los 14 ó 15 años de escolaridad con una certificación validada y reconocida por el mercado laboral, quien a su vez es capaz de recompensar salarialmente dicha calificación, es pertinente con determinados sectores laborales más dinámicos y en expansión. Es posible preveer en este caso inserciones laborales más regulares y relativamente estables, con mejores posibilidades de contar con empleos con mayor reconocimiento social, de ascenso social en el sector de activi- 
dad, acceder a las formalidades laborales, seguridad social, perfeccionamiento y nuevas calificaciones.

iv) Trayectorias de tipo profesionales/académicas. Quienes alcanzan los 16 ó 17 años de escolaridad, equivalentes a la formación completa de educación superior en sus diversas modalidades. Este colectivo, siendo minoritario en la población juvenil chilena, pero con una fuerte y sostenida tendencia a la expansión en las últimas décadas, logra encontrar una ubicación relativamente permanente y mejorada en el mercado laboral y salarial.

En los jóvenes, la transición de la escuela al trabajo o hacia la continuidad de estudios superiores ha sido siempre un momento crítico en la construcción de un plan de vida. Esta problemática se complejiza en la actualidad, pues los años de escolarización ya no garantizan un empleo y, si bien se siguen solicitando credenciales o licencias educativas, otros factores vinculados con el capital social y cultural resultan —a veces - muy definitorios para la inserción laboral y educacional. Nos enfrentamos, entonces, al fenómeno genérico que las transiciones de los jóvenes de hoy, a lo menos, se caracterizarían por ser largas y complejas.

En el caso de las trayectorias de trabajo, el proceso de inserción se ha extendido en el tiempo. Se alternan períodos de desocupación, empleos precarios, pasantías y/o becas y estudios antes de lograr una cierta estabilización en el empleo, si es que ésta llega. En el caso de los estudios, los proyectos parecen estar más definidos, pero se encuentran, generalmente, supeditados a la obtención de un empleo que permita costearlos, por supuesto, también se renuevan los cuestionamientos hacia la propia biografía y las opciones y elección de un tipo de proyecto de vida.

Proyecto de vida e identidad, son dos variables que nos aparecen en el contexto del estudio y que se encuentran en una relación dialéctica, pues cada uno contribuye a la formación del otro. La constitución de la identidad favorece y permite la definición y consolidación de un proyecto de vida. Este proceso se encuentra a su vez en interacción con el contexto sociocultural, que aportará discursos, valores, significaciones, modelos, para la elaboración de los contenidos de la identidad del YO y de sus proyectos de vida.

El proyecto de vida, entonces, se establece sobre un futuro que se desea alcanzar y sobre un conjunto de representaciones de lo que no está todavía, pero se desea lograr. Es decir, se apoya sobre representaciones del presente y del pasado, sobre un conocimiento, 
sobre una interpretación determinada y sobre una cierta selección de hechos pasados y presentes, a la luz de una intención futura. La elaboración representativa de la situación presente orienta la construcción del proyecto y, supone una cierta reflexión sobre las estrategias para llevarlo a cabo y sobre los motivos que lo sostienen.

Crear un proyecto significa poder utilizar los soportes y recursos que fueron brindados para la realización de algo propio, algo en lo cual el sujeto se reconozca. La definición del proyecto de vida puede tomarse como indicador del trabajo intrasubjetivo que ha realizado el sujeto en relación a la construcción de su identidad. Luego, poder construir un proyecto implica realizar una historización de las experiencias vitales, incorporando la categoría de futuro.

Bien sabemos que las transiciones vitales de adolescencia y/o juventud pueden provocar situaciones de angustia frente a lo nuevo. La identidad se ve amenazada por la transformación que acontece en estos períodos y, la transición de la escuela al trabajo, necesariamente ubica al sujeto en relación a un futuro incierto, en tanto desconocido, nuevo. Crear un proyecto de vida/vital requiere evaluar estas situaciones críticas para poder elegir entre distintas alternativas de estilos de vida.

El sujeto, entonces, necesitará enfrentar las crisis determinadas por las transiciones vitales, evolutivas y socioculturales y, la manera en que las resuelva dependerá de sus recursos personales y del apoyo de las redes sociales: familia, escuela, trabajo, política social, en donde éstas podrán funcionar tanto como soporte de las posibilidades de cambio, como obstáculo de las mismas.

El itinerario de vida del mundo juvenil está cambiando, la llamada moratoria social o proceso de transición de la niñez a la adultez, se ha interrumpido como experiencia para un número importante de nuestros jóvenes. Hasta ahora la tradición de vida cotidiana y de superación de las metas de movilidad social, efectivamente se daban a través del estudio, pero de manera lineal o continúa. Esta experiencia que resultaba cómoda en tanto establecía un claro itinerario a seguir y conseguir en la perspectiva de asumir el rol o estatus de adultos, ya no tiene la relevancia de antes, pues los jóvenes cada vez más, producto de la incertidumbre de sus itinerarios de vida, se ven provocados a combinar roles de jóvenes y de adultos que antes eran impensable para el desarrollo de la juventud en la sociedad chilena.

Esto efectivamente tiene que ver con las transformaciones sociales, económicas y políticas que sufren las sociedades y que afecta no sólo a los jóvenes como grupo social, sino que en general a los 
pobres o a las personas que frente a los cambios y transformaciones societales aparecen con menos ventajas sociales o menos oportunidades integracionales en la perspectiva del capital individual y el capital social que portan para ganar nuevas y mejores posiciones en el sistema social de cara a esas transformaciones.

Educación y trabajo, entonces, pasan a ser parte fundamental en el trazado de los itinerarios de vida que dibujan y escogen los jóvenes de hoy, que en su calidad de estudiante proyectarán para toda su vida adulta. El tema no es menor, frente a la incertidumbre de itinerarios tradicionalmente lineales y continuos, nos aparecen o nos podrían aparecer, cada vez más itinerarios diversos y diversificados y, por lo tanto, los jóvenes efectivamente correrán una vez más el riesgo de no acertar correctamente y quedar socialmente excluidos. Conocer de ello con anticipación, es decir, conocer el capital individual que portan, el capital cultural de que disponen, el apoyo que le proporciona la familia, las oportunidades o restricciones referidas al género o a la procedencia, y las opciones que el sistema social y políticos está ofreciendo, es tarea necesaria y urgente en la perspectiva de acompañar a estos sujetos juveniles en el proceso de su individualización, en el proceso de construcción de un proyecto de vida/vital.

\section{FACTORES PERSONALES E INSTITUCIONALES INCIDENTES EN LAS TRAYECTORIAS EDUCACIONALES Y LABORALES}

Los ámbitos educativos y laborales se pueden identificar y analizar como componentes diferenciados, pero en la vida del sujeto, ambos se presentan imbricados, potenciándose uno a otro. Ello quiere decir que en el orden más formal y convencional, primero viene el tiempo de estudiar y luego el tiempo de trabajar, entendiéndose que se estudia para aprender a trabajar; y más dinámicamente hay tiempos de trabajo que generan procesos formativos. Todas estas experiencias son asumidas como las deseables y esperables para los jóvenes, en su relación con el mundo del estudio y el trabajo.

En los itinerarios de vida de las personas se van acumulando recursos que pueden condicionar la posibilidad de construcción de los proyectos vitales de los sujetos, repotenciando las mismas trayectorias educacionales y laborales. Estos recursos se van generando de una manera dinámica en la relación del sujeto con las diversas experiencias y espacios sociales en los cuales se desenvuelve. Para el caso de nuestro estudio hemos diferenciado en factores institucionales y facto- 
res personales. Los institucionales son los referidos al sistema educacional, sistema laboral, la familia y la política social, asumidos como los elementos sociales que van aportando diversos recursos a los sujetos durante su vida. Los factores personales, los hemos asumido como el sí mismo, los recursos individuales, entendido como el capital individual en trabajo y estudio, y el capital social. En las historias de vida de los jóvenes estos factores se presentan con distintos grados de intensidad. Desde el punto de vista metodológico hemos establecido tres niveles: alto, medio y bajo, dependiendo del grado de acumulación que representan en su biografía personal. A partir de ello, se determina el nivel de incidencia de estos factores como elementos que pueden potenciar mayores posibilidades de ascender y proyectarse en la trayectoria educacional y laboral de cada individuo.

A continuación se describe las trayectorias educacionales y laborales de once (11) jóvenes, construido a partir de sus historias de vida. En estos casos se analizan la incidencia que tienen los factores personales e institucionales en las trayectorias educacionales y laborales a partir de la actividad social que desempeñan en tiempos actuales.

\section{a) No estudia ni trabaja}

\begin{tabular}{|c|c|c|c|c|c|c|}
\hline \multicolumn{7}{|c|}{ HOMBRE, 16 AÑOS: TRAYECTORIA LINEAL } \\
\hline Educación & Laboral & Familia & $\begin{array}{c}\text { Política } \\
\text { social }\end{array}$ & Sí mismo & $\begin{array}{c}\text { Recursos } \\
\text { personales }\end{array}$ & $\begin{array}{c}\text { Capital } \\
\text { social }\end{array}$ \\
\hline Bajo & Bajo & Mediano & Bajo & Bajo & Bajo & Bajo \\
\hline
\end{tabular}

En este caso se presenta una trayectoria corta. Su relación con los factores institucionales es precaria. Posee sólo siete años de escolaridad, experiencias laborales de carácter informal y ocasionales, sin vínculos con la política social. La relación con la familia opera básicamente en el plano de la subsistencia, sin mayores influencias y soportes para alentar su permanencia en el sistema escolar.

Ello influye que los factores personales también aparezcan con un bajo nivel de incidencia. Ha acumulado escasos recursos educacionales y laborales; su capital social también es muy escaso contando sólo con relaciones sociales reducidas a su círculo de amigos del barrio en su localidad, que están en su misma condición de desvinculación de la escuela. En el sí mismo cuenta con escaso nivel de reconocimiento de habilidades; desde el punto de vista de sus expectativas tiene más presente el trabajar y el retornar a la escuela es parte de sus 
aspiraciones, asignándole una valoración muy alta respecto a lo que puede significar en la vida de un sujeto, pero poco cercana a sus posibilidades. Su ventana de ausencia del mundo escolar es muy corta, lo que le permitiría retomar su itinerario educacional, clave para su desarrollo, siendo el factor política social y educacional, determinante para su reinserción educacional.

\begin{tabular}{|c|c|c|c|c|c|c|}
\hline \multicolumn{7}{|c|}{ MUJER 20 AÑOS: TRAYECTORIA SINCRONIZADA } \\
\hline Educación & Laboral & Familia & $\begin{array}{c}\text { Política } \\
\text { social }\end{array}$ & Sí mismo & $\begin{array}{c}\text { Recursos } \\
\text { personales }\end{array}$ & $\begin{array}{c}\text { Capital } \\
\text { social }\end{array}$ \\
\hline Mediano & Bajo & Mediano & Bajo & Mediano & Mediano & Mediano \\
\hline
\end{tabular}

En este segundo caso, los factores institucionales si bien aparecen con una incidencia mediana, cursa hasta primer año medio y el clima educacional y laboral es positivo en su familia de origen, no son suficiente para mantener su presencia en el sistema educacional.

Laboralmente desempeña trabajos sin calificación y no encuentra trabajo estable. No tiene vínculo con la política social. En el ámbito de los factores personales, dispone de un mediano capital social, sólo a nivel de amigos, familia y relaciones sociales diversas, pero que no alcanzan a nivel institucional, que le permita resolver sus necesidades de trabajo, de continuidad de estudios u otros beneficios sociales. En sus recursos y capital individual, el más significativo es su escolaridad. A nivel del sí mismo si bien reconoce habilidades y tiene expectativas y posibilidades de continuar estudiando, su problema mayor está en el plano de las necesidades materiales para la subsistencia.

\begin{tabular}{|c|c|c|c|c|c|c|}
\hline \multicolumn{7}{|c|}{ MUJer 20 AÑOs: TRAYECTORIA «YO-YO» } \\
\hline Educación & Laboral & Familia & $\begin{array}{c}\text { Política } \\
\text { social }\end{array}$ & Sí mismo & $\begin{array}{c}\text { Recursos } \\
\text { personales }\end{array}$ & $\begin{array}{c}\text { Capital } \\
\text { social }\end{array}$ \\
\hline Mediano & Bajo & Mediano & Mediano & Bajo & Mediano & Mediano \\
\hline
\end{tabular}

La presencia de factores institucionales aparece con un nivel de incidencia mediano, básicamente por el acumulado que implica el tipo de trayectoria que desarrolla. En lo educativo deserta en la enseñanza básica, pero vuelve a hacer la enseñanza media en la modalidad de educación de adulto obteniendo certificación de cuarto año medio laboral, que no le permite continuidad de estudios terciarios. Es una joven que tiene una incidencia mediana en la política social pues, cuenta con apoyo para ella y sus niños que se encuentran asistiendo a 
jardín infantil. Su familia opera como soporte en la subsistencia y acogida para su núcleo familiar. Lo laboral se presenta bajo, pues el tipo de trabajo que desempeña no tienen calificación, es informal y ocasional. En los factores personales, en lo referido a capital social, su vinculación institucional, le otorga posibilidades de resolver algunas necesidades de continuidad de estudios y capacitación laboral. Su escolarización y capacitación la dota de capital individual, en lo educacional y laboral. El factor más resentido es el sí mismo, en cuanto sus expectativas están más bien a un nivel aspiracional. Sin duda que el factor laboral, es el más determinante para la posibilidad de mejorar sus posibilidades de desarrollo, en la medida que pudiera generarle mejores condiciones materiales para iniciar nuevos emprendimientos.

\section{b) Sólo estudia}

\begin{tabular}{|c|c|c|c|c|c|c|}
\hline \multicolumn{7}{|c|}{ HOMBRE 29 AÑOS: TRAYECTORIA «YO-YO» } \\
\hline Educación & Laboral & Familia & $\begin{array}{c}\text { Política } \\
\text { social }\end{array}$ & Sí mismo & $\begin{array}{c}\text { Recursos } \\
\text { personales }\end{array}$ & $\begin{array}{c}\text { Capital } \\
\text { social }\end{array}$ \\
\hline Mediano & Mediano & Mediano & Alto & Alto & Mediano & Alto \\
\hline
\end{tabular}

Este joven ha logrado configurar una nueva situación a partir de haber consolidado una segunda etapa de su ciclo escolar. Aquí se da una relación también directa entre factores institucionales y factores personales, pero de acuerdo a la biografía del sujeto, es una relación más dinámica en la cual los factores personales juegan un rol decisivo. La alta valoración de sí mismo, el capital social acumulado, fruto de procesos de vinculación y participación social y política, son los que le permiten generar las relaciones para su subsistencia, acceder a la educación de adultos y terminar su enseñanza media.

La incidencia media de sus recursos educacionales y laborales, se relacionan con el hecho que aunque valora la certificación de egresado de enseñanza media, siente que no le garantiza acceder a la universidad, al mismo tiempo incide la ausencia de una calificación laboral que le permita un trabajo estable. Es un sujeto con altas expectativas que se podrían materializar a partir de los recursos con que cuenta. Lo laboral es lo que aparece con la dimensión más resentida y que será decisiva para su posibilidades futuras. 


\section{c) Sólo trabaja}

\begin{tabular}{|c|c|c|c|c|c|c|}
\hline \multicolumn{7}{|c|}{ HOMBRE, 23 AÑOS: TRAYECTORIA LINEAL } \\
\hline Educación & Laboral & Familia & $\begin{array}{c}\text { Política } \\
\text { social }\end{array}$ & Sí mismo & $\begin{array}{c}\text { Recursos } \\
\text { personales }\end{array}$ & $\begin{array}{c}\text { Capital } \\
\text { social }\end{array}$ \\
\hline Alto & Bajo & Mediano & Alto & Bajo & Alto & Mediano \\
\hline
\end{tabular}

En este caso se presenta una situación singular. Es un joven con una trayectoria estrictamente lineal, ha desarrollado toda su vida escolar únicamente dedicado a los estudios, ha tenido una educación de calidad, recorriendo todo el itinerario de manera continúa, acumulando un alto capital individual, en términos de recurso educacionales y laborales, ha cursado los tres niveles de la educación. Sin embargo, ese proceso no le ha permitido hasta este momento resolver su dilema principal, dotarse de las bases materiales para el sustento de él y su familia.

El único factor institucional deficitario es el laboral, en tanto su vinculación es al egreso de la universidad, de carácter ocasional y en labores sin calificación. Su familia de origen ha colaborado en todo su trayecto educativo y aún en el presente es un soporte para ayudar a sostener a su núcleo familiar. La singularidad que se presenta es que esta significativa incidencia de los factores institucionales no cualificaron los factores personales con la misma significación. Su capital social es escaso, su espectro de relaciones se reducen a su familia y amigos del barrio y ha tenido esporádica relación con la política social como el crédito estudiantil.

El sí mismo se ve afectado, en cuanto existe un cúmulo de necesidades materiales, su núcleo familiar que depende de él y su pareja, su nivel de expectativas y estado de ánimo está bajo y deprimido. Aquí las modificaciones para posibilitar un buen desarrollo de su proyecto vital sin duda pasan por factores más bien subjetivos y una conexión adecuada con la política social en materia de información y orientación.

\section{d) Estudia y trabaja}

\begin{tabular}{|c|c|c|c|c|c|c|}
\hline \multicolumn{7}{|c|}{ HOMBRE, 18 AÑOS: TRAYECTORIA SINCRONIZADA } \\
\hline Educación & Laboral & Familia & $\begin{array}{c}\text { Política } \\
\text { social }\end{array}$ & Sí mismo & $\begin{array}{c}\text { Recursos } \\
\text { personales }\end{array}$ & $\begin{array}{c}\text { Capital } \\
\text { social }\end{array}$ \\
\hline Mediano & Mediano & Mediano & Bajo & Alto & Alto & Mediano \\
\hline
\end{tabular}


Este joven desde los inicios de la enseñanza media combina los estudios con trabajos remunerados. La relación con el mundo del trabajo ha tenido un desarrollo incremental, de trabajos sin calificación a trabajos calificado, trabajos ocasionales a más estables, el elemento formalidad es el que ha estado ausente. Su itinerario educacional ha sido continuo, con un buen rendimiento académico, el oficio que estudia, gastronomía, ha incidido en una mejor vinculación con el mundo del trabajo, empata con la tradición familiar y opera como base para sus aspiraciones, estudiar cocina internacional en la educación terciaría.

La experiencia social, principalmente la laboral, le ha dotado de un significativo capital social, permitiéndole logros en sus aspiraciones laborales. Su familia ha estado presente colaborando con estas experiencias laborales y motivando su escolarización. Hasta la fecha no presenta ningún vínculo con la política social. Respecto a los factores personales, su sí mismo aparece alto, en cuanto no presenta grandes necesidades, tiene una visibilidad y valoración de sus habilidades, conciencia de sus aprendizajes sociales y altas expectativas. En consideración con su proyecto de educación terciaria su capital individual se presenta alto. Desde el punto de vista de las posibilidades de viabilizar sus aspiraciones lo que aparece más ausente es el apoyo de la política social.

\begin{tabular}{|c|c|c|c|c|c|c|}
\hline \multicolumn{7}{|c|}{ HOMBRE, 22 AÑOS: TRAYECTORIA SINCRONIZADA } \\
\hline Educación & Laboral & Familia & $\begin{array}{c}\text { Política } \\
\text { social }\end{array}$ & Sí mismo & $\begin{array}{c}\text { Recursos } \\
\text { personales }\end{array}$ & $\begin{array}{c}\text { Capital } \\
\text { social }\end{array}$ \\
\hline Alto & Alto & Mediano & Alto & Alto & Alto & Alto \\
\hline
\end{tabular}

Este joven se encuentra cursando la educación terciaria, está en segundo año de la carera técnico en matricería de plásticos y metales. Su trayectoria se vuelve sincronizada a partir de su egreso de la enseñanza media, cuando desarrolla la práctica laboral. Desde ahí cursa la universidad estudiando y trabajando. Su relación con el mundo laboral es desempeñando oficios calificados y en relaciones contractuales formales, acomodando el desempeño de un oficio de manera estable, compatible con sus tiempos de estudios.

Su itinerario educacional es continuo, desempeñándose como un buen alumno en los ciclos básicos y medios. Su familia, opera como un soporte efectivo en relación a su inserción en la universidad. Tiene una relación con la política social, en tanto cuenta con crédito 
universitario. Su sí mismo se presenta alto, ya que no tiene mayores necesidades, cuenta con expectativas y motivaciones para terminar la carrera y reconoce sus habilidades. Respecto a su capital individual, sus recursos educacionales y laborales, son altos. Al igual su capital social, toda vez que le permite atender sus temas laborales, de soportes y apoyos estudiantil.

\begin{tabular}{|c|c|c|c|c|c|c|}
\hline \multicolumn{7}{|c|}{ MUJER, 22 AÑOS: TRAYECTORIA SINCRONIZADA } \\
\hline Educación & Laboral & Familia & $\begin{array}{c}\text { Política } \\
\text { social }\end{array}$ & Sí mismo & $\begin{array}{c}\text { Recursos } \\
\text { personales }\end{array}$ & $\begin{array}{c}\text { Capital } \\
\text { social }\end{array}$ \\
\hline Alto & Mediano & Mediano & Bajo & Alto & Alto & Alto \\
\hline
\end{tabular}

Esta joven también inicia su trayectoria sincronizando estudio y trabajo a partir de los 18 años, es decir, en el momento del egreso de la enseñanza media. Realiza su itinerario educacional básico y medio sin mayores problemas. De igual modo tiene un buen desempeño en la universidad, donde se encuentra en tercer año de enfermería. La familia ha sido un soporte principal en cuanto la ha alentado e invertido en su educación y aún hasta hoy financia en parte sus estudios. Su relación con lo laboral ha sido en trabajos sin calificación y temporales, se desempeña de manera más estable en labores de aseo de oficinas.

La opción de trabajar es para sustentar su proyecto educacional. No dispone de ningún tipo de apoyo de la política social. Los factores personales se presentan óptimos en tanto tiene un cierto capital, producto de su vinculación con su iglesia evangélica y, de sus relaciones construidas en lo laboral. El sí mismo también se presenta alto, reconoce sus habilidades, tiene expectativas altas y no presenta necesidades materiales. Sus recursos educacionales son altos al igual que los laborales. El único elemento que atentaría contra el desarrollo de su proyecto, sería su desvinculación con la política social, en tanto su familia pudiera no seguir otorgando soporte económico a sus estudios.

\begin{tabular}{|c|c|c|c|c|c|c|}
\hline \multicolumn{7}{|c|}{ MUJER, 28 AÑOS: TRAYECTORIA SINCRONIZADA } \\
\hline Educación & Laboral & Familia & $\begin{array}{c}\text { Política } \\
\text { social }\end{array}$ & Sí mismo & $\begin{array}{c}\text { Recursos } \\
\text { personales }\end{array}$ & $\begin{array}{c}\text { Capital } \\
\text { social }\end{array}$ \\
\hline Alto & Alto & Mediano & Alto & Alto & Alto & Alto \\
\hline
\end{tabular}

Esta joven inicia su trayectoria sincronizada durante la enseñanza media, alrededor de los 16 años de edad. Su itinerario educativo es largo y lo realiza sin grandes dificultades, se considera una buena alumna en rendimiento académico: la básica, un liceo técnico profe- 
sional, el instituto técnico profesional y la universidad, sumando ya 17 años de escolaridad. Hasta ahora la valoración y opción de trabajar tiene que ver con sustentarse su proyecto más personal. Anhela estudiar algo más cercano a su vocación de trabajo.

Su relación con la política social se materializa a través de beca Presidente de la República, hasta la enseñanza media. En el orden de los factores personales tiene una alta percepción de su capital individual. Su sí mismo incide altamente, no tiene actualmente grandes necesidades materiales, tiene una alta valoración de sus habilidades y altas expectativa acerca de su futuro. Estamos ante una trayectoria educacional y laboral plenamente desarrollada donde esta joven sólo se plantea formarse para su plenitud laboral y vocacional, en condiciones que ya tiene resuelta su autonomía personal.

\begin{tabular}{|c|c|c|c|c|c|c|}
\hline \multicolumn{7}{|c|}{ HOMBRE, 28 AÑOS: TRAYECTORIA SINCRONIZADA } \\
\hline Educación & Laboral & Familia & $\begin{array}{c}\text { Política } \\
\text { social }\end{array}$ & Sí mismo & $\begin{array}{c}\text { Recursos } \\
\text { personales }\end{array}$ & $\begin{array}{c}\text { Capital } \\
\text { social }\end{array}$ \\
\hline Alto & Alto & Mediano & Bajo & Alto & Alto & Alto \\
\hline
\end{tabular}

Inicia su trayectoria sincronizada entre los 14 y 15 años de edad cursando el segundo año medio. En los últimos años vive un constante proceso de capacitación y toma diversos cursos de especialización que puedan actualizarlo y potenciar su posicionamiento laboral.

Su relación con lo laboral se inicia en labores sin calificación, luego se desempeña en trabajos que exigen calificación, siempre de manera estable $\mathrm{y}$ en relaciones contractuales formales. La familia aporta medianamente como un factor en tanto su relación es más independiente de ella desde temprana edad. Su relación con la política social sólo se establece en el momento que es internado en un liceo por la aguda situación familiar que presenta.

El capital social y los recurso educacionales acumulados en el internado y en el mundo del trabajo aparecen como decisivos en la biografía de esta persona. Estamos en presencia de una trayectoria educacional y laboral plenamente desarrollada, con un sujeto posicionado en el mundo laboral y ciclos continuos de educación y formación; una persona que ha consolidado su autonomía. Su proyecto vital está signado por la necesidad de seguir perfeccionándose y actualizándose en lo formativo y laboral. 


\begin{tabular}{|c|c|c|c|c|c|c|}
\hline \multicolumn{7}{|c|}{ MUJER, 25 AÑOS: TRAYECTORIA SINCRONIZADA } \\
\hline Educación & Laboral & Familia & $\begin{array}{c}\text { Política } \\
\text { social }\end{array}$ & Sí mismo & $\begin{array}{c}\text { Recursos } \\
\text { personales }\end{array}$ & $\begin{array}{c}\text { Capital } \\
\text { social }\end{array}$ \\
\hline Alto & Alto & Mediano & Alto & Alto & Alto & Alto \\
\hline
\end{tabular}

Ella inicia su trayectoria sincronizada a los 14 años de edad. Su itinerario educacional se presenta continuo y de la educación media pasa a un instituto profesional donde se recibe de técnico en diseño y planificación social y, posteriormente estudia un magíster. Su relación con lo laboral se da desde temprana edad a partir de trabajos no calificados, adquiere el oficio de garzona, se va ubicando en trabajo más calificados pero ocasionales, para posteriormente, a medida que avanza su educación técnica y profesional, desarrollar trabajo especializados y estables.

Nuevamente son los factores personales, el capital social acumulado en las tempranas experiencias laborales y el sí mismo, traducido en altas expectativas, reconocimiento de habilidades y capacidades, los que se vuelven decisivos en el desarrollo de la biografía de esta joven. También estamos aquí ante una trayectoria laboral plenamente desarrollada, con una experiencia laboral consolidada, con un nivel de educación superior completo y, con una autonomía personal ya conquistada. Un sujeto con expectativas de buscar nuevos espacios de desarrollo laboral y educacional más acorde a su vocación y necesidad de formación.

Las trayectorias educacionales y laborales, analizadas en el amplio espectro que estas historias de vida permite, sirven para comprender cómo los sujetos reaccionan o se adaptan ante las circunstancias que rodean el itinerario y desarrollo de su proyecto vital en camino hacia la vida adulta.

Todos estos jóvenes tienen como signo común pertenecer a hogares de escasos recursos económicos, en su mayoría hijos de padres con oficios no calificados y calificados, sin formación profesional. Sin embargo, los destinos en curso y finales van por rumbos distintos. ¿Qué hace esta diferencia de trayectorias en sujetos que, en términos generales, tienen un mismo punto de partida? Si bien las trayectorias educacionales y laborales que hemos presentado tienen como primer propósito sólo caracterizar el recorrido, sin pretender establecer de manera concluyente razones y causalidades, sí puede entregar elementos que permitan explicar estas diferencias. 
La gran mayoría ha usado una trayectoria educacional y laboral del tipo sincronizada, siete jóvenes, esto quiere decir que en el desarrollo de su itinerario educacional, al mismo tiempo han estado trabajando remuneradamente. En las historias de vida que estamos analizando la relación educación y trabajo tiene distintas significaciones y consecuencias para estos sujetos. Los jóvenes que han iniciado su actividad laboral en la enseñanza media, dejan claro que a lo menos no lo hicieron por necesidad de subsistencia. Tampoco declaran que lo hacen por sostener económicamente su educación media. Lo relevante de este dato es que la única persona que trabaja por razones de subsistencia, cursando el octavo año básico, abandona su itinerario educacional en segundo año medio.

$\mathrm{Y}$ quienes sincronizan las actividades de estudio y trabajo, lo hacen con el consentimiento y apoyo de sus padres. Podemos señalar que son padres, que sin embargo le asignen valor a la educación y, no valorizan negativamente que sus hijos trabajen y estudien al mismo tiempo. De este grupo de jóvenes, tres de ellos han desarrollado el ciclo educacional completo a nivel de educación terciaria, con 16 años y más de escolaridad. Son jóvenes que además costean autónomamente su educación técnica y profesional. El cuarto caso transita aún por la educación media técnico profesional.

Dos jóvenes iniciaron su trayectoria sincronizada al terminar la enseñanza media para colaborar a financiar parte de sus estudios. Ambos dependen de sus padres para el desarrollo de su carrera profesional y ésta aún se encuentra en curso.

Dos jóvenes desarrollan un tipo de trayectoria educacional y laboral del tipo «yo-yo», esto quiere decir que primero hubo una etapa de estudio, luego un período de trabajo y luego volvieron a estudiar. Ambos recibieron igual certificación pero de distintas calidades, que frente al futuro para reiniciar otro período de estudio, los coloca en desiguales condiciones. Ambos terminaron la enseñanza media, una obtuvo una certificación de enseñanza media que sólo sirve para el mundo el trabajo, y la otra obtuvo una certificación que le permite la continuidad de estudios en el nivel terciario. Los efectos en las subjetividades de los sujetos son claramente diferenciadas. Uno, se anima y está expectante con su apuesta de llegar a la universidad, con sus 29 años y, la otra persona, con sus 20 años, ve interrumpida sus aspiraciones de continuidad de estudios.

Por último, dos jóvenes desarrollaron su trayectoria educacional y laboral del tipo lineal. El primero de ellos, sin embargo, la inte- 
rrumpió en el séptimo básico y, hoy a sus 16 años, se ve desprovisto de un capital individual y social. Su única posibilidad de continuidad de estudios es su reinserción en el sistema de educación de adultos.

El segundo caso de trayectoria lineal, cursó todos los niveles de educación —incluida la terciaria—, no desarrollando experiencias en el mundo del trabajo. Hoy en día tiene serias dificultades de encontrar trabajo en su especialidad. Paradójicamente, este último joven, a pesar de haber estudiado en una universidad una carrera técnica, no cuenta con estrategias de búsqueda o generación de empleo. Lo relevante de esta situación es que a pesar de haber hecho todo su itinerario formativo en un continuo en la institucionalidad educativa, y de haber mostrado una gran adaptabilidad a los requerimientos del mundo de la educación, no dispone de igual capacidad de adaptabilidad al mundo del trabajo.

Lo medular que entregan estos antecedentes es que las experiencias de vida de los jóvenes están siguiendo trayectorias diversas. En esas trayectorias los factores institucionales y personales se mueven y afectan de manera también diversas las biografías los sujetos. A la luz de estos elementos, se puede levantar algunas reflexiones más generales.

La educación es el principal vehículo de movilidad social y es el factor decisivo para mejorar la calidad de vida de las personas. Así como afecta positivamente en capital individual de los sujetos, no garantiza una formación integral que asegure herramientas de adaptabilidad que permitan a los sujetos desempeñarse en los diversos espacios sociales. Una gran adaptabilidad al sistema educacional no implica una gran adaptabilidad a los requerimientos de la vida.

El mundo del trabajo, tiene distintos significados para los jóvenes, diferentes ventajas y desventajas, el sistema educacional debe aprender a reconocerlos, y hacer esfuerzos por asumir esos significados. Los jóvenes ingresan al mundo del trabajo por distintas razones y/o necesidades. En este estudio hemos logrado identificar cuatro dimensiones asociadas lo anterior: i) la dimensión de subsistencia, asociado a su condición económica y social; ii) la dimensión experiencial, existe el deseo de experimentar el trabajo, participar del mundo laboral y el estatus que ello implica, en tanto reconocimiento social y garantías para disponer de recursos propios; iii) la dimensión instrumental, como soporte que permite sostener sus proyectos de vida más deseados, como lo es el proyecto educacional; y, iv) la dimensión vocacional, como un estado o lugar de realización personal y profesional. 
Estas diversas dimensiones no están vedadas o excluidas para los jóvenes con menores ventajas sociales. Sus necesidades materiales, la cultura del trabajo de sus padres, la dificultad para financiar su educación, son motivos presentes que pueden llevan a asumir en algún momento la experiencia del mundo del trabajo. Los costos que ello implica no son deseables, como el abuso y no cumplimiento de sus derechos y garantías individuales y legales, excesivo desgaste y agotamiento cuando se estudia y trabaja, baja en los niveles de rendimiento académico.

Al mismo tiempo, existen significativas ganancias, más allá de lo pecuniario, en lo formativo, los jóvenes que trabajan desarrollan habilidades y procesos de maduración relevantes que influyen en su desarrollo individual y social. Una de las dificultades en el sistema educacional es la rigidez en su pedagogía, normas y tiempos que no le permiten ser un espacio flexible para este tipo de experiencia. El sistema educacional se flexibiliza, pero sólo a nivel de la educación terciaria y en la modalidad de educación de adulto.

La familia es otro factor institucional relevante que afecta el itinerario de los jóvenes estudiantes. De acuerdo a la realidad social de estos jóvenes, sus familias pueden jugar roles en función del discurso formativo de animar y de motivar a sus hijos a adquirir mayor educación para alcanzar mayores grados de movilidad social; la función de orientar y normar en el cumplimiento de las exigencias escolares; $y$, la función de invertir con mayores recursos en la educación de sus hijos. La desvalorización del poco capital educacional de la familia, de los padres en particular, adquiridos en otras épocas, impiden un mayor aporte de la familia. Sin embargo, los jóvenes reconocen y valoran la preocupación e inversión que hacen sus padres para apoyar sus proyectos educacionales.

La política social, en particular la dimensión de los recursos y soportes para fortalecer la permanencia de los jóvenes en el sistema educacional, la intervención institucionalizada de la orientación y el apoyo personalizado a los estudiantes, dentro del liceo o en la institucionalidad comunal, es el gran ausente en las historias de vida de estos jóvenes. Es escasa la ocasión en que se presenta durante el desarrollo de la educación media y, se hace más presente cuando los jóvenes ya se encuentran en la educación terciaria. Cuando ésta se hace presente tiene un gran nivel de incidencia en el fortalecimiento de los procesos de continuidad de sus itinerarios. 
La posibilidad de conocer y comprender las trayectorias educacionales y laborales de los jóvenes estudiantes, en particular de los que están en situación de pobreza, y cómo se conjugan los factores que están incidiendo en ellas, pueden facilitar una intervención que permita modificar sus proyectos vitales. Como afirmamos, estas trayectorias desde el punto de vista de los sujetos están develando parte de sus estrategias de ascenso, pero también el comportamiento rígido que las instituciones de la política social están desempeñando en su relación con los sujetos.

\section{LOS JÓVENES QUE SÓLO ESTUDIAN Y LOS JÓVENES QUE ESTUDIAN Y TRABAJAN A LA VEZ}

Las representaciones sociales involucran una construcción de sentido y cierta actitud. La historia familiar, escolar y laboral, construida a través de los procesos de socialización primaria y secundaria, va configurando esas representaciones, que inciden en los proyectos de los jóvenes. La construcción biográfica condensa elementos de significación para comprender su perspectiva acerca de los horizontes educacionales y laborales. Desde las representaciones de los adolescentes, el estudio y el trabajo se constituyen en vertebradores de los proyectos de vida. Éstos supuestamente habilitan para la formulación de estrategias necesarias para su implementación.

Para los jóvenes la continuidad de estudios es uno de los objetivos más claros. El trabajo se hará necesario como medio para sostener el proyecto educativo que se plantean. Mientras que el estudio es percibido como un itinerario posible, la inserción laboral se encuentra presente en las argumentaciones de los adolescentes como necesidad. Más allá de la necesidad que expresan de conseguir un empleo que permita desarrollar un proyecto educativo futuro, encuentran obstáculos para lograr una inserción al mundo del trabajo. A la crisis vital, se le suman las restricciones impuestas por el contexto social y productivo que dista de garantizarles un empleo.

De esta forma encontramos una situación paradojal: mientras que para los jóvenes las habilitaciones se hacen posibles a través del trabajo, las dificultades crecientes para obtenerlo inciden en las posibilidades de construcción de sus proyectos. Hasta hace pocos años los jóvenes se planteaban en su elección, al finalizar la educación media, opciones de estudio o de trabajo. En la actualidad, para muchos jóvenes, el trabajo es condición necesaria para poder estudiar. Pero la posibilidad de lograr un empleo se hace cada vez más lejana, en función de la restricción estructu- 
ral del contexto. Asimismo, las precarias condiciones laborales existentes para quienes logran una inserción laboral —en cuanto a horarios, rotación, bajas remuneraciones - inciden en la posibilidad de hacer coexistir el trabajo con el estudio, lo que obstaculiza la realización de la meta vinculada con la continuidad de un proyecto educacional.

El trabajo también va a significar para los jóvenes un medio que se visualiza como eficaz para lograr independencia. Debemos tener en cuenta que este objetivo resulta especialmente relevante en este momento vital. Por lo tanto, sostendrá no sólo proyectos educacionales sino también otros proyectos personales.

Los jóvenes se encuentran en una situación en la que necesitan llegar a una elección, tomar una decisión, y esto produce un conflicto que se refleja en la tensión entre lo «que me gusta y lo que me conviene». La posibilidad de continuar estudios, y de tener un rol activo en la construcción de un itinerario educacional y laboral se encuentra lesionada: por un lado, las elecciones están mediadas por la necesidad de contar con cierta garantía de inserción laboral, y por otro, se complejiza la posibilidad de conjugar estudio y trabajo.

Los jóvenes perciben una mayor exigencia académica respecto de la situación vivida en la escolaridad media. El conocimiento de los planes de estudios de las carreras en las que manifiestan interés es difuso, y se constatan representaciones estereotipadas de roles ocupacionales. Más allá que visualicen un recorrido educacional futuro, la falta de información provoca inseguridad e incide en la movilización de estrategias para transitar por esos itinerarios. De esta forma, aún visualizando las metas posibles, la situación descrita obstaculiza un rol activo en la arquitectura de las propias trayectorias educacionales.

Los resultados de nuestra investigación nos han permitido identificar a lo menos tres tipos de trayectorias o itinerarios de vida que correlacionan o no las actividades sociales de estudio y trabajo. Ellos son, trayectorias lineales, trayectorias sincronizadas y trayectorias «yo-yo». Estos tipos de trayectorias no tienen valor en la lógica del estudio, las tres pueden ser buenas formas de alcanzar o sustentar los proyectos personales de los jóvenes, todo dependerá de cómo operan o se comportan los factores personales y sociales que portan los individuos y de los movimientos que ellos generen, ascendentes, provocando más y mejores cambios en las inserciones educacionales y laborales, o descendentes, provocando regresiones o retrocesos en sus inserciones laborales y educacionales. El cuadro resumen que a continuación presentamos, es el resultado de un análisis pormenorizado de 
los grupos de estudiantes involucrados, es decir, aquellos que sólo estudian o aquellos que sincronizan la actividad de estudio y trabajo en la enseñanza media, en la educación de adultos y en los centros de formación técnica y, su relación con cuatro variables significantes: los recursos individuales, el capital social, sus expectativas en el plano de la educación y el trabajo y el proyecto de vida futuro. El comportamiento de esas variables dio por resultado tipos de trayectorias que son las que se presentan y explican a continuación como parte del resultado de este estudio.

Cuadro Resumen

Tipos de trayectorias, según grupo de estudiantes

\begin{tabular}{|l|c|c|c|c|c|c|}
\hline $\begin{array}{l}\text { Tipo de grupo } \\
\text { de joven }\end{array}$ & $\begin{array}{c}\text { Recursos } \\
\text { individuales }\end{array}$ & $\begin{array}{c}\text { Capital } \\
\text { social }\end{array}$ & Expectativa & $\begin{array}{c}\text { Estado } \\
\text { de ánimo }\end{array}$ & Proyecto & $\begin{array}{c}\text { Tipo } \\
\text { trayectoria }\end{array}$ \\
\hline $\begin{array}{l}\text { Sólo estudia en } \\
\text { la enseñanza } \\
\text { media regular. }\end{array}$ & Altos & Bajo & Media & Positivo & $\begin{array}{c}\text { Difuso en } \\
\text { educación } \\
\text { y trabajo }\end{array}$ & Lineal \\
\hline $\begin{array}{l}\text { Estudia y } \\
\text { trabaja en la } \\
\text { enseñanza } \\
\text { media regular. }\end{array}$ & Medios & Alto & Alta & $\begin{array}{c}\text { Muy } \\
\text { positivo }\end{array}$ & $\begin{array}{c}\text { Claro en } \\
\text { educación } \\
\text { y trabajo }\end{array}$ & $\begin{array}{c}\text { Sincroni- } \\
\text { zado }\end{array}$ \\
\hline $\begin{array}{l}\text { Sólo estudia en } \\
\text { la educación de } \\
\text { adultos. }\end{array}$ & Bajos & Bajo & Baja & Positivo & $\begin{array}{c}\text { Claro en } \\
\text { estudio } \\
\text { difuso en } \\
\text { trabajo }\end{array}$ & Lineal \\
\hline $\begin{array}{l}\text { Estudia y } \\
\text { trabaja en la } \\
\text { educación de } \\
\text { adultos. }\end{array}$ & Altos & Alto & Alta & $\begin{array}{c}\text { Muy } \\
\text { positivo }\end{array}$ & $\begin{array}{c}\text { Claro en } \\
\text { trabajo y } \\
\text { estudio }\end{array}$ & «yo-yo» \\
\hline $\begin{array}{l}\text { Sólo estudia en } \\
\text { un centro de } \\
\text { formación } \\
\text { técnica. }\end{array}$ & Medios & Medio & Media & Positivo & $\begin{array}{l}\text { Claro en } \\
\text { trabajo, } \\
\text { difuso en } \\
\text { estudio }\end{array}$ & Lineal \\
\hline $\begin{array}{l}\text { Estudia y } \\
\text { trabaja en un } \\
\text { centro de } \\
\text { formación } \\
\text { técnica. }\end{array}$ & Altos & Medio & Alta & Muy \\
positivo & $\begin{array}{c}\text { Claro en } \\
\text { educación } \\
\text { yrabajo }\end{array}$ & Sincroni- \\
zado
\end{tabular}

a) Trayectorias lineales

Las trayectorias lineales se caracterizan por establecer relaciones de causa-efecto, son etápicas, es decir, un antes precede a un después y los eventos principales de la vida de una persona nunca se pre- 
sentan de manera simultánea porque los espacios y ámbitos de la vida están socialmente organizados por distintas instituciones sociales. Ésta ha sido la manera tradicional de entender los procesos de transición o de moratoria de nuestros jóvenes, en tanto se enfatiza la adquisición de capacidades, habilidades y derechos asociados a la vida adulta, proceso que se desarrolla principalmente mientras se es estudiante. El liceo, entonces, cumple la función de colaborar con el tránsito de los jóvenes de una etapa de vida a otra, en este caso de la etapa de estudiante a la etapa de trabajador.

En nuestro estudio, y en la realidad chilena, esta lógica de entender a los jóvenes y su proceso de desarrollo se sigue manteniendo para un número considerable de jóvenes. En el caso que estamos analizando, el $62 \%$ de los estudiantes de enseñanza media, el 56.5\% de los jóvenes que están en la educación de adultos y el $68 \%$ de los jóvenes que estudian en un centro de formación técnica, se encuentran en esta situación. Lo relevante del dato es que la institucionalidad educacional ya no acoge en sus centros educacionales a un $100 \%$ de jóvenes que sólo ejercen la actividad del estudio y que se preparan para seguir posteriormente otras etapas de vida, trabajar, independizarse, formar su propia familia. El fenómeno que se nos presenta es que la transición de los jóvenes a la vida adulta, ya no sólo no es lineal, sino que tampoco es un sólo tipo de transición.

El cuadro anterior da cuenta de esta situación, aquellos que se ubican en la lógica de transiciones lineales, son un grupo de jóvenes que durante su educación básica, educación media y pos-secundaria han desarrollado sólo la actividad social del estudio. Poseen distinto tipo de capital individual cultural, alto, medio y bajo, medido en función de su desempeño y/o rendimiento escolar y de las experiencias de fracaso vividas durante la educación básica o media. Tienen un mediano o bajo capital social, es decir, bajo reconocimiento de habilidades sociales. Presentan una expectativa mediana o baja respecto de sus posibilidades de estudio y/o trabajo, sin embargo, presentan un positivo estado de ánimo futuro. Sus proyecto de vida futuro asociado al estudio o al trabajo se presenta difuso en las dos áreas o difuso en una de las dos áreas, en educación o en trabajo.

El desarrollo de este tipo de trayectoria tendrá dos movimientos absolutamente predecibles, el ascendente o el descendente. Observamos que en los tres casos de grupos de jóvenes que manifiestan la tendencia a desarrollar trayectorias lineales, que el factor asociado al capital individual cultural, será determinante en la definición de sus 
proyectos de vida/vital. Por lo tanto, mientras mayor capital individual aporten, mayor posibilidad de provocar movimientos ascendentes que resuelvan adecuadamente la construcción o superación de sus metas educacionales y laborales y que les permita adquirir nuevas posiciones sociales. A la inversa, mientras menos capital individual cultural, más posibilidades de provocar movimientos descendentes, descontinuando su trayectoria lineal o estableciendo rupturas o regresiones con altas consecuencia negativas para la inserción social.

\section{b) Trayectorias sincronizadas}

Las trayectorias sincronizadas, son un fenómeno relativamente nuevo en nuestra realidad chilena. Dice relación con el desarrollo de dos o más ámbitos de la vida que se despliegan de manera simultánea en la etapa de vida juvenil o en la etapa de vida adulta. Estamos refiriéndonos concretamente al desarrollo simultáneo de las actividades de estudio y trabajo. Este fenómeno dice relación con factores económicos y con factores socioculturales que comienzan a vivir nuestra sociedad y que impacta en el desarrollo de la vida de nuestros jóvenes, provocando una superposición de las etapas de vida. Hoy en día se puede, efectivamente, ser estudiante y jefe de hogar, trabajador y vivir con los padres o estudiante y trabajador a la vez, o estudiar y ser padre o madre.

El fenómeno concreto de ser estudiante y trabajador a la vez mientras se es adolescente o joven, es un hecho social que, como lo hemos indicado anteriormente, era impensable en la lógica tradicional de entender la preparación de nuestros jóvenes a la vida adulta. Existía en el imaginario social, la necesidad de salvaguardar la trayectoria de vida de los jóvenes protegiéndola del ejercicio de roles de adultos, en tanto no estaban preparados. El trabajo en menores o en adolescentes de hecho era considerado - y aún lo es - un atentado a los derechos de la niñez y la juventud, fundamentalmente porque la actividad laboral, generada al margen de las leyes sociales no protegía y no protege efectivamente sus derechos.

Se observa, entonces, que el factor económico, en familias y jóvenes de escasos recursos económico influyó notoriamente en el desarrollo de este tipo de trayectoria. Efectivamente, muchas familias de escasos recursos económicos han debido aceptar el trabajo infantil o adolescente de sus propios hijos en función de superar las crisis económicas familiares o individuales. Pero también se observa que 
existe un fenómeno cultural que deviene de la prolongación de la juventud, en donde los jóvenes de distintos estratos sociales, en promedio atraviesan las diferentes etapas de la vida a una mayor edad: finalizan los estudios más tarde de lo establecido, acceden al trabajo más tarde, se casa a edades más superiores o crean su familia en promedios de edades superiores a lo conocido en nuestra tradición social. Es decir, en muchos casos estamos observando que la relación estudio y trabajo, también tendría una relación de carácter más instrumental en tanto permite a los sujetos sostener efectivamente el desarrollo de sus proyectos de vida personal más deseados.

En el caso de este estudio aquellos que se ubican en el tipo de trayectorias denominadas sincronizadas, son un grupo de jóvenes que durante la educación media y en la educación superior desarrollan de manera simultánea las actividades de estudio y trabajo y que desean mantener este tipo de trayectoria en el desarrollo de sus proyectos futuros. Son jóvenes que en general presentan un buen capital individual, medido en rendimiento escolar y ausencia de experiencia de fracaso escolar, aunque hay que indicar que el desempeño escolar en los jóvenes de la educación media regular se hace más difícil, pues la institucionalidad escolar, en general, no tiende a apoyar y valorar positivamente este tipo de experiencia. Son jóvenes, que además presentan altas expectativas de desarrollo de sus oportunidades educacionales y laborales, manifestando un muy positivo estado de ánimo. Sus proyectos de vida futuro tanto en el plano educacional como en el laboral se perciben claramente, pero mucho más en la necesidad de alcanzar o resolver sus metas educacionales y, lo laboral operando como factor que favorecería ese logro. En ambos grupos de jóvenes que delinean este tipo de trayectorias, se aprecia un alto capital social, que deviene justamente de su experiencia laboral.

En este caso los movimientos que puede generar este tipo de trayectoria también son dos, ascendente o descendente. Observamos, que en la medida que los jóvenes que estudian y trabajan en la enseñanza media regular logren mantener o aumentar su capital individual cultural, es mucho más posible que generen movimientos ascendentes, que puedan favorecer el logro de mejores posiciones de inserción social.

c) Trayectorias «yo-yo»

Las trayectorias «yo-yo» (López, 2002; Machado, 2002), es otra opción que deviene de los fenómenos descritos anteriormente, y 
que tiene seguramente una mayor ocurrencia en ciertos niveles educacionales y laborales de la población joven y adulta. Se diferencia de la anterior en el sentido que son tipos de trayectorias que si bien no superponen las actividades de estudio y trabajo, funcionan como requisito una de la otra en forma permanente. Es decir, es el tipo de trayectoria de aquellas personas que primero estudian, luego trabajan y luego vuelven a estudiar tantas veces como los requerimientos de sus proyectos personales o factores externos así lo determinen. A la rigidez de las transiciones lineales, se ha pasado también a una nueva situación en la que los jóvenes construyen y vuelven a construir sus biografías siguiendo los vaivenes de la vida y tratando de influir en sus resultados.

En el caso de este estudio, observamos que tiene dos sentidos importante. Por un lado, el desarrollar este tipo de trayectoria permite a los jóvenes alcanzar en forma más pausada y progresivas sus metas educacionales a través del trabajo. Es decir, alternar períodos de estudio con períodos de trabajo, tiene un carácter más instrumental, en tanto sirve para costear lo que más se desea, alcanzar metas educacionales, es un tipo de trayectoria que también es motivada o influenciada por las realidades económicas que les toca vivir a estos sujetos. Por otro lado, podría tener directa relación con la especialización laboral y con los requerimientos que la actividad laboral va demandando, sobre todo en el caso que se constituya en una exigencia de su trabajo o empleo y que le permite alternar períodos de estudio o especialización con períodos de trabajo. Estaría más asociado a lo que hoy en día el Programa Chile Califica, está denominando educación permanente.

Aquellos que se ubican en el tipo de trayectorias «yo-yo», son un grupo de jóvenes que estudia en la educación de adultos. Son jóvenes que abandonaron la enseñanza media regular porque tuvieron que trabajar, durante la educación media adulto sincronizan la actividad de estudios, pero en su proyecto futuro, preferirían alternar períodos de trabajo con estudio, hasta lograr estudiar lo que más desean. Poseen un alto capital cultural individual y un alto capital social, como así mismo un muy positivo estado de ánimo frente al futuro. Sus proyectos futuros se presentan claros y definidos.

Los movimientos que puede generar este tipo de trayectoria son ascendente o descendente. Y, observamos, que el gran riesgo de este tipo de trayectoria es que los períodos de alternancia pueden ser muy largos, y desalentar el ir y venir, tal como un «yo-yo», el desarrollo de las actividades estudio, trabajo, estudio, para provocar cambios 
en la curva ascendente de su trayectoria. El peligro, entonces, es que efectivamente la alternancia puede provocar discontinuidades, retrocesos e inseguridades en los sujetos respecto de la consistencia de sus proyectos personales y las formas de cómo alcanzarlos.

Si hubiese que establecer un juicio acerca de las posibilidades de estos tipos de trayectorias de vida, los datos están indicando que aquellas trayectorias que son del tipo sincronizada, para este tipo de estudiante, aparece como una posibilidad más exitosa. La conjugación de variables tan determinantes como lo es el capital cultural que portan y el capital social que poseen, augura mejores perspectivas integracionales.

Observamos que el capital cultural, su acumulación y utilidad frente a las opciones de vida que barajan los jóvenes, es una función muy importante que debe cumplir el liceo con un mayor nivel de calidad, pero al mismo tiempo también observamos que siendo ella —el liceo-, una de las principales instituciones socializadoras de los jóvenes, en la mayoría de los casos el capital social, es más bien adquirido extra la experiencia educacional. He ahí un interesante reflexión a desarrollar, en tanto efectivamente, los jóvenes constituyen la imagen del «liceo» a partir de las significaciones sustentadas en su percepción sobre las relaciones de saber y la construcción de vínculos que tienen lugar en la realidad cotidiana escolar. La realidad cotidiana del liceo es el lugar más próximo en el cual los jóvenes se manifiestan y debemos comprender que estas percepciones son también tributarias de un orden más estructural, ligado en este caso a la normatividad de nuestro propio sistema educativo.

Desde esta perspectiva nos interrogamos en qué medida las instituciones escolares y educacionales conciben su proyecto institucional, y qué propósitos, contenidos y prácticas específicas las guían. Al mismo tiempo que preguntarse por cuáles son los proyectos de los jóvenes y en qué medida el liceo o el centro de formación técnica está verdaderamente interesada en ellos. Si el liceo no integra a los jóvenes sobre la base del reconocimiento, sólo queda un escenario que dista de sentar condiciones favorables para el desarrollo de la identidad personal. Si el liceo se vacía de conocimientos significativos para los jóvenes, y si la problemática vital de éstos no es tomada en cuenta, se desaprovecha un espacio de gran potencialidad para fortalecer la construcción de la subjetividad. Pues no se trata sólo de habilitarlos correctamente para estudiar o para trabajar, se trata de la posibilidad de asentar la identidad, la individualidad, la biografía de estos sujetos y 
con ello la capacidad de sostener en el tiempo sus propios proyectos personales.

Intentando reconocer algunas variables de carácter estructural, la imagen que podemos desarrollar es la de jóvenes secundarios, en especial los adscritos a establecimientos municipalizados, es que en su dimensión de estudiantes tienden a compatibilizar la actividad de estudio con el trabajo, ya sea de manera esporádica - temporada de verano - o permanente. De la misma manera, se puede establecer que se trata de jóvenes que se encuentran en una situación socioeconómica precaria. Este factor es relevante, por cuanto la condición de pobreza, por lo demás sobredimensionada por las autoridades escolares, es asociada mecánicamente a situaciones de riesgo social (Oyarzún et al., 2001).

Esta situación posee particular importancia, ya que mirado desde una perspectiva estrictamente sociológica, la enseñanza media municipalizada en Chile, se ha convertido en el sistema educativo de los pobres. Ello porque la sostenida universalización de la enseñanza básica detonó, ya a comienzos de la década de 1980, la crisis de la segunda enseñanza, la cual no logró readecuar los sentidos de la educación frente a su nuevo escenario social. De acuerdo con Eduardo Castro, la enseñanza media ya no puede operar con criterio elitarios y discriminatorios, ya que con su $90 \%$ de cobertura se ha convertido en la enseñanza de la clase trabajadora (Castro, 1992).

En este plano, la condición de pobreza de los jóvenes que acceden a la educación municipalizada se convierte en factor de negación de sus potencialidades culturales. Como señala Lutte, los estudiantes de las clases populares, cuya cultura difiere de la cultura escolar, deben realizar esfuerzos considerables para asimilarla sometiéndose a una reeducación de la misma (Lutte, 1991:174).

Cabe preguntarse, entonces, ¿qué es lo que hoy día hace la diferencia? Sin lugar a dudas no es la condición de pobreza en sí. Pero sí habría que asumir que la llegada masiva de los jóvenes pobres hasta los establecimiento secundarios detonó transformaciones importantes, no sólo en la composición de la matrícula secundaria, sino que fundamentalmente en la forma en la cual comenzaron a relacionarse los sujetos al interior del sistema escolar. Los jóvenes pobres se convirtieron, tanto desde el punto de vista de las estrategias escolares, como desde la perspectiva de las relaciones de administrativas, en una figura compleja, difícil de aceptar, reconocer y de asumir.

Habría que preguntarse también si se puede hablar con propiedad, que para este sector de jóvenes, sus posibles inserciones futuras por 
la vía educacional, se encuentran canceladas, teniendo que buscar otras vías de inserción diferentes a las de escolarización formal, habida cuenta de la ausencia de políticas y programas de protección y asistencia social desde el sistema institucional que pudiesen inscribirse en la línea de hacer reversible esta etapa y no entenderla como un ciclo terminal y determinante. Da la impresión que en Chile, la realidad que vive todavía una proporción significativa de jóvenes, pudiendo incluirse a ciertos sectores medios precarizados, sigue con plena vigencia la concepción conceptual de los itinerarios juveniles de tipo lineal $-\mathrm{y}$ en este caso, trayectorias fallidas-, más que las trayectorias reversibles, laberínticas o del tipo «yo-yo» que nos habla Machado Pais (López, 2002).

El abordaje de los trayectos o itinerarios de educación y trabajo en jóvenes, que cursan o están por finalizar o han finalizado la educación media y transitan por la educación pos secundaria, debería permitirnos entonces, la comprensión desde la perspectiva de los actores acerca de las implicancias de los contextos macro-sociales, y por otro lado, desde una mirada más micro-social, los factores que operan condicionando las posibilidades y limitaciones, para el diseño o arquitectura de los propios proyectos de vida/vital.

Nuestra focalización temática, en torno a la educación y el trabajo en la estructuración de identidades y su papel en la construcción de proyectos de vida, no fue casual. La educación y el trabajo se constituían en factores estructuradores de identidades absolutamente predecibles, estables y permanentes, hasta los años 80 . Hoy las transformaciones de la economía y el trabajo con su correlato en las transformaciones del conocimiento y la ciencia, sumado a las crisis sociolaborales, produce cambios significativos en la manera tradicional de entender los procesos de transición, construcción de identidad y posicionamiento de los sujetos en la sociedad, siendo el elemento central de este cambio, la emergencia de procesos de transición que se desarrollan en contexto de incertidumbre.

En esta lógica los sistemas de educación no están al margen de los efectos que provocan las transformaciones macro-sociales. Por un lado, es observable que el sistema de educación está afectado por su propia crisis a nivel de sus sentidos y orientaciones, la que a su vez impacta en la desestructuración de las biografías en los sujetos, e interroga sobre las posibilidades de su integración social. Por otro lado, es evidente que la profundización de la segmentación educativa, por circuitos diferenciados en el sistema escolar, impregnan las representaciones y resienten los proyectos y metas de los sujetos. 
La selección de las poblaciones estudiadas tampoco fue casual, ya que permite abordar distintas etapas vitales claves en la necesidad de significar y orientar y/o reorientar los proyectos de vida. Nuestro análisis se ha dispuesto iluminar en qué medida la visualización de los recursos personales con que cuentan los jóvenes, condicionan la movilización de estrategias que permitan alcanzar los proyectos que se proponen como metas.

Los ámbitos de socialización y las instituciones sociales tienen un papel protagónico como dispositivos protectores u obstaculizadores para los mismos. Desde este lugar planteamos que el liceo debe y puede cumplir una tarea decisiva en el proceso de construcción de sentido, de conocimiento significativo y de desarrollo de los jóvenes. El liceo se constituye en un lugar controvertido para los jóvenes, a la que se demanda y cuestiona, pero también habilita un espacio privilegiado de integración social, en el encuentro con pares y adultos extrafamiliares.

Visualizar los recursos personales, también es un factor decisivo en las actitudes de los jóvenes. Desde nuestro enfoque, la transición estudio-empleo no se puede concebir sólo como el problema de la inserción laboral de un sujeto que posee ciertas competencias, sino como un proceso complejo de construcción de la identidad. Definir un proyecto de vida/vital moviliza un proceso de construcción de significaciones, conduce a interrogarse sobre sí mismo, su identidad, su entorno, sus recursos, posibilidades y obstáculos; aparecen entre los mismos jóvenes diversas posibilidades de transición y desarrollo de sus trayectorias de vida, algunos con más oportunidades y otros que no logran fortalecer y/o reconocer sus propios proyectos vitales.

Desde el liceo, los sistemas de orientación pueden ayudar a preparar a los jóvenes para las múltiples transiciones que deberán enfrentar en su vida. Fortalecer la identidad personal y cultural, estimular la formación, desarrollar los recursos personales: autoconfianza, sentido crítico, autonomía, valores éticos y mejorar su habilidad para resolver problemas complejos, así como realizar las elecciones para su inserción socio-laboral. Puede además, ayudar a retener a los sujetos en el sistema educativo, para elevar sus recursos y evitar la exclusión laboral y social. La orientación, los sistemas de orientación educacional, deben contribuir a enfrentar estas necesidades, en tanto dispositivo capaz de articular educación y trabajo en el sistema educacional. Pues, si se debilita la construcción de recursos personales e individuales y el liceo no contribuye a sentar condiciones favorables 
para fortalecer la autoestima y asumir una actitud reflexiva y crítica, el futuro de las personas profundiza en inseguridad. Acompañar al sujeto en la construcción de un proyecto que tome en cuenta su historia y su experiencia cultural, así como el conocimiento y relación con su entorno, es una necesidad para tomar una decisión personal que le permita ampliar sus alternativas.

De esta manera la política educacional a tiempos actuales, de acuerdo a los modos en que la población juvenil está constituyendo sus itinerarios de vida, le compete no sólo anhelar una mejor inclusión de sus jóvenes al sistema social sino, que sobre manera buscar las estrategias que permita materializar y concretar tal aspiración.

VIÑA DEL MAR, ABRIL 2003

\section{BIBLIOGRAFÍA}

BELLEI, CRistián (2000): «Educación media y juventud en los 90. Actualizando la vieja promesa». Última Década Nº12. Viña del Mar: Ediciones CIDPA.

BEYER, HARALD (2000): «Educación y desigualdad de ingresos: una nueva mirada». Estudios Públicos N ${ }^{\circ} 77$. Santiago: CEP.

BIZQUERRA, RAFAEL (1990): Orientación psicopedagógica para la prevención y el desarrollo. Madrid: Praxis.

CASTRO SiLVA, EDUARDO (1992): «La educación media y los retos de la modernidad». Documento de Trabajo N56. Santiago: CPU.

CEPAL-UNESCO (1992): Educación y conocimiento: eje de la transformación productiva con equidad. Santiago: CEPAL-UNESCO.

CERRI, MARIANELA (1989): «Rendimiento escolar y trabajo remunerado en alumnos de enseñanza básica y media». En JUAN E. GARCÍA-HUIDOBRO (editor): Escuela, calidad e igualdad. Los desafíos para educar en democracia. Santiago: CIDE.

COMISIÓN NACIONAL PARA LA MODERNIZACIÓN DE LA EDUCACIÓN (CNME), (1995): Los desafíos de la educación chilena frente al siglo XXI. Informe de la comisión nacional para la modernización de la educación. Santiago: Editorial Universitaria.

DÁVILA LEÓN, OSCAR (2002): «Biografías y trayectorias juveniles». Última Década Nº17. Viña del Mar: Ediciones CIDPA. 
DELORS, JACQUES (1997): La educación encierra un tesoro. Informe a la UNESCO de la comisión internacional sobre la educación para el siglo XXI. París: Correo de la UNESCO.

DU BOIS-REYMOND, MANUELA (2002): «Transiciones modernizadas y políticas de desventaja: Países Bajos, Portugal, Irlanda y jóvenes inmigrantes en Alemania». Revista de Estudios de Juventud $\mathrm{N}^{\circ} 56$. Madrid: INJUVE.

GORDILLO, MARÍA VICTORIA (1996): Orientación y comunidad. La responsabilidad social de la orientación. Madrid: Alianza.

LÓPEZ BLASCO, A. (2002): «De los itinerarios lineales a las trayectorias 'yo-yo'». Ponencia presentada en la Conferencia Europea para Investigadores y Técnicos «Jóvenes y políticas de transición en Europa», INJUVE, Madrid.

LUTTE, G. (1991): Liberar la adolescencia. La psicología de los jóvenes de hoy. Barcelona: Editorial Herder.

MACHADO PAIS, JosÉ (2002a): «Laberintos de vida: paro juvenil y rutas de salida (jóvenes portugueses)». Revista de Estudios de Juventud N56. Madrid: INJUVE.

MidePLAN (2001): «Situación de la educación en Chile 2000». Santiago: MIDEPLAN.

MoRCH, M. (2002): «Sistemas educativos en sociedades segmentadas: 'trayectorias fallidas' en Dinamarca, Alemania Oriental y España». Revista de Estudios de Juventud Nº56. Madrid: INJUVE.

OSORIO ROJAS, R.: «Orientación educacional y educación integral». En www.nodo50.org.

OYARZÚN, ASTRID et al. (2001): Entre jóvenes re-productores y jóvenes co-constructores: sentidos de la integración en la cultura escolar. Viña del Mar: Ediciones CIDPA.

REDONDO ROJO, JESÚs (1997): «La dinámica escolar: de la diferencia a la desigualdad». Revista de Psicología Vol. VI. Santiago: Universidad de Chile.

RODRÍGUEZ, SEBASTIÁN (1998): «La función orientadora. Claves para la acción». Revista de Investigación Educativa, 16 (2).

ROSSETTI, JOSEFINA (2003): Diagnóstico sobre la orientación y el rol socio-profesional de los orientadores en los establecimientos educacionales chilenos. Santiago: PIIE.

TEDesco, JuAn CARLOS (2000): Educar en la sociedad del conocimiento. Buenos Aires: FCE. 